\title{
Patient, Provider, and Clinic Characteristics Associated with Opioid and Non-Opioid Pain Prescriptions for Patients Receiving Low Back Imaging in Primary Care
}

\author{
Laura S. Gold, PhD, Zachary A. Marcum, PharmD, PhD, Eric N. Meier, MS, \\ Judith A. Turner, PhD, Kathryn T.James, PA, MPH, David F. Kallmes, MD, \\ Patrick H. Luetmer, MD, Brent Griffith, MD, Karen J. Sherman, PhD, \\ Janna L. Friedly, MD, MPH, Pradeep Suri, MD, Richard A. Deyo, MD, MPH, \\ Sandra K. Johnston, PhD, Andrew L. Avins, MD, MPH, Patrick J. Heagerty, PhD, and \\ Jeffrey G. Jarvik, $M D, M P H$
}

Background: To describe characteristics of patients, providers, and clinics associated with opioid or non-opioid pain medication prescribing patterns for patients who received lower spine imaging in primary care clinics.

Methods: In these secondary analyses of the Lumbar Imaging with Reporting of Epidemiology (LIRE) study, a randomized controlled trial conducted in 4 health systems in the United States, we evaluated characteristics associated with receipt of pain medication prescriptions. The outcomes were receipt of prescriptions for opioid or, separately, non-opioid pain medications within 90 days after imaging. Among patients who received opioid or non-opioid prescriptions, we evaluated receipt of multiple prescriptions in the year following imaging. Mixed models were used to estimate adjusted odds ratios (ORs) and 95\% confidence intervals (CIs).

Results: Compared with whites, patients identified as Asian (OR, 0.53; 95\% CI, 0.51-0.56), Native Hawaiian/ Pacific Islander (OR, 0.73; 95\% CI, 0.64-0.83), multiracial (OR, 0.84; 95\% CI, 0.71-0.98) or Black (OR, 0.92; 95\% CI, 0.89-0.96) had significantly reduced odds for receiving prescriptions for opioids within 90 days. Patients identified as Native American/Alaska Native had greater odds for receiving prescriptions for non-opioid pain medications within 90 days $(0 R, 1.12 ; 95 \%$ CI, 1.01-1.24). Receipt of pain prescriptions 120 days before imaging was strongly predictive of subsequent receipt of pain prescriptions across all categories.

Conclusions: After adjusting for factors that could affect prescribing, the strongest differences observed in pain-medication prescribing were across racial categories and for patients with previous pain prescriptions. Further research is needed to understand these differences and to optimize prescribing. $(\mathrm{J} A m$ Board Fam Med 2021;34:950-963.)

Keywords: Back Pain, Diagnostic Imaging, Drug Prescriptions, Non-0pioid Analgesics, Opioid Analgesics, Primary Health Care, Race Factors

\section{Introduction}

Low back pain is a complex condition with many possible etiologies. ${ }^{1}$ Treatments include medications,

This article was externally peer reviewed.

Submitted 26 January 2021; revised 16 April 2021; accepted 19 April 2021.

From the Department of Radiology, School of Medicine, University of Washington, Seattle, WA (LSG, KTJ, SKJ, JGJ); Clinical Learning, Evidence, and Research Center, University of Washington, Seattle, WA (LSG, ENM, JAT, KTJ, JLF, PS, SKJ, PJH, JGJ); Department of Pharmacy, School of Pharmacy, University of Washington, Seattle, WA (ZAM); Department of Biostatistics, University of Washington, Seattle, WA (ENM, $\mathrm{PJH})$; Department of Psychiatry and Behavioral Sciences, University of Washington, Seattle, WA (JAT); Department of Rehabilitation Medicine, University of Washington, Seattle, WA (JAT, JLF); Department of Radiology Mayo Clinic, Rochester, MN (DFK, PHL); Department of Radiology, Henry Ford Hospital, Detroit, MI, (BG); Kaiser Permanente complementary and alternative medicine (CAM) treatments, psychological therapies, interventional therapies (eg, injections), and spine surgeries. ${ }^{2}$

Washington, Seattle, WA (KJS); Rehabilitation Care Services, VA Puget Sound Health Care System, Seattle, WA (PS); Departments of Family Medicine and Internal Medicine, Oregon Health \& Science University, Portland, OR (RAD); Division of Research, Kaiser Permanente Northern California, Oakland, CA (ALA).

Funding: This work was supported within the National Institutes of Health (NIH) Health Care Systems Research Collaboratory by the NIH Common Fund through cooperative agreement U24AT009676 from the Office of Strategic Coordination within the Office of the NIH Director and cooperative agreements UH2AT007766 and UH3AR066795 from the National Institute of Arthritis and Musculoskeletal and Skin Diseases (NIAMS). Research was completed by investigators at the University of Washington Clinical Learning, Evidence and Research (CLEAR) Center for Musculoskeletal Disorders 
Although prescription opioid medications are generally not recommended ${ }^{3-6}$ and such use has decreased in recent years, ${ }^{7,8}$ they are still often prescribed for low back pain; in 2016, opioids were prescribed to $20 \%$ of patients after new encounters for low back pain in a large, population-based study. ${ }^{8}$ Non-opioid medications are also often prescribed for low back pain and include muscle relaxants, nonsteroidal antiinflammatory drugs (NSAIDs), gabapentinoids, tricyclic antidepressants (TCAs), and serotonin-norepinephrine reuptake inhibitors (SNRIs). ${ }^{4,9}$

Patterns of opioid prescribing vary by patient racial/ethnic characteristics, with Blacks and Hispanic/Latinx patients less likely than whites to receive opioid prescriptions. ${ }^{10-13}$ Some evidence indicates that patients living in lower socioeconomic status (SES) neighborhoods may be more likely to receive opioid prescriptions for new back pain episodes than patients in higher SES neighborhoods. ${ }^{14}$ Other patient characteristics associated with variations in opioid prescribing include age, with middleaged patients receiving more opioid prescriptions for back pain from primary care providers (PCPs) compared with those $>60$ years or $<30$ years, and insurance type, with Medicaid or self-pay patients receiving more opioid prescriptions than those with private insurance. ${ }^{15}$ Relationships between patient sex and opioid prescribing have been examined in previous studies, with some reporting that male patients are less likely to be prescribed opioids compared with female patients ${ }^{16-18}$ and others reporting no differences by patient sex. ${ }^{15,19}$

Clinic characteristics are also likely associated with opioid prescribing. Patients who receive care in rural clinics are more likely to receive prescriptions for opioids compared with patients who receive care in urban areas. ${ }^{20}$ Opioid-related mortality rates have increased more rapidly in rural

and supported by the National Institute of Arthritis and Musculoskeletal and Skin Diseases (NIAMS) of the National Institutes of Health under Award Number P30AR072572. The content is solely the responsibility of the authors and does not necessarily represent the official views of the National Institutes of Health. This work is registered at ClinicalTrials.gov identifier: NCT02015455.

Conflict of interest: In the spirit of full disclosure, we have several potential conflicts of interest to report. JAT reports receiving royalty payments from Psychological Assessment Resources, Inc (PAR). DFK holds patients related to spine augmentation and has licensed intellectual property related to spine augmentation from the Mayo Clinic. RAD reports receiving royalties from Wolters Kluwer/UpToDate for authoring topics on low back pain and consulting fees from Kaiser Permanente Washington Health Research Institute, than in urban areas, and prescription opioids are more commonly involved than heroin or synthetic opioids in drug-related deaths in rural areas, suggesting that opioids are more readily prescribed in rural locations. ${ }^{21}$

Little is known about provider characteristics associated with opioid prescribing. A study in 2 military emergency department facilities found that physician assistants were more likely than physicians to have prescribed opioids. No differences in prescribing rates between male and female providers were found. ${ }^{22}$

Increasing knowledge regarding the characteristics of patients, providers, and clinics associated with the prescription of opioid or non-opioid medications for back pain may point to opportunities for optimizing care that is consistent with clinical guidelines. This secondary analysis aimed to describe patient-level, provider-level, and cliniclevel characteristics associated with pain medication prescribing for patients who received low back imaging in the primary care setting to generate hypotheses for future studies. Because our sample size was large, we described patterns of pain medication prescribing in understudied patient populations such as patients in relatively small racial categories. In addition, because we had access to a wide variety of patient, provider, and clinic characteristics, we could model associations adjusting for previously identified trends and report on novel associations that future work can examine.

\section{Methods}

\section{Study Design and Data Sources}

The parent study for these secondary analyses was the Lumbar Imaging with Reporting of Epidemiology (LIRE) study, described previously in detail. ${ }^{23,24}$ The rationale of the LIRE study was that lumbar spine imaging often uncovers findings that are unrelated to pain and if providers were

as well as partial salary support from an endowment from Kaiser Permanente. JGJ reports royalties as a book co-editor from Springer Publishing and travel reimbursement for Faculty Board of Review from GE-Association of University Radiologists Radiology Research Academic Fellowship (GERRAF) and royalties as a chapter author from Wolters Kluwer/UpToDate.

Corresponding author: Laura S. Gold, $\mathrm{PhD}$, Department of Radiology, School of Medicine, University of Washington, 4333 Brooklyn Ave NE Box 359558, Seattle, WA, USA 98195-9558, Phone: 206-543-2749, Fax: 206-543-8609 (Email: goldl@uw.edu). 
aware of how often these findings seem in patients who do not have back pain, they might be less alarmed by them and thus less likely to recommend potentially unnecessary interventions. Primary care clinics within 4 large health care systems [Mayo Clinic (Rochester, Minnesota); Henry Ford (Detroit, Michigan); Kaiser Permanente Northern California (Oakland, California); and Kaiser Permanente Washington (Seattle, Washington)] were randomly assigned to generate imaging reports containing epidemiologic benchmark text containing the prevalence of common imaging findings among patients (with or without back pain) at varying start dates. ${ }^{24-26}$ Before the intervention, clinics received imaging reports without the intervention text. Data were obtained from comprehensive electronic medical records (EMR) that captured health care utilization, including prescription medication data.

Within each health system, clinics that provided primary care for adult patients were identified. Patients $\geq 18$ years old who received lower spine imaging from October 1, 2013, to September 30, 2016, who had no spine imaging within the past year and had not opted out of research studies were automatically enrolled in the trial. LIRE providers were defined as PCPs whose main practices were at 1 of these clinics and who ordered at least 1 qualifying spine image during the trial.

The LIRE study found no differences in the primary outcome, spine-related health care utilization in the year following the index lumbar spine image and found no differences in spine-related health care utilization in the year following the index spine imaging, among patients whose images did versus did not contain the intervention text. However, patients whose images contained the intervention text had a small but statistically significant decrease in the likelihood of receiving prescriptions for opioids within a year of their index images. ${ }^{23}$

For this secondary analysis, all participating institutional review boards determined that the study was minimal risk and granted waivers of consent and Health Insurance Portability and Accountability Act (HIPAA) authorization.

\section{Prescription Drug Identification}

Opioid and non-opioid pain medications were identified by a pharmacist (Supplemental Table 1) using drug information databases (IBM Micromedex ${ }^{\circledR}$ and $\left.\mathrm{UpToDate}^{\circledR}\right)$. Non-opioid medication classes that we examined were skeletal muscle relaxants, NSAIDs, gabapentinoids, tricyclic antidepressants, and benzodiazepines. We also included the only SNRI that had Food and Drug Administration (FDA) approval for treatment of chronic musculoskeletal pain, duloxetine, in the non-opioid pain medication category. We included oral and topical forms and excluded pain medication prescriptions that occurred during inpatient stays and medication forms used exclusively or nearly exclusively in inpatient settings. We identified pre-index imaging pain medication prescriptions as those that occurred in the 120 days before the date of the spine image, and post-imaging prescriptions were those that occurred from the date the index image was finalized by the interpreting radiologist (hereafter, this date is termed "index") through 90 days later. Preimaging medications included outpatient prescriptions written by any provider but, because we were interested in prescribing trends among PCPs, postimaging prescriptions were only those from LIRE providers. However, unless otherwise stated, the provider who wrote prescriptions did not have to be the same 1 who ordered the index image if both met the definition of LIRE providers. We analyzed ordered, not necessarily filled, prescriptions because we were interested in prescribing trends. Prescriptions written between the image date and the finalized image report were not counted as pre-imaging or postimaging prescriptions. Nonprescription pain medications were not captured in the EMR and are not included in these analyses.

\section{Patient, Provider, and Clinic Variables}

We evaluated the characteristics of patients who received opioid or non-opioid pain medication prescriptions. Patient-level characteristics were obtained from the EMR and included age (categorized as 18 to $39 ; 40$ to 60 , and $\geq 61$ years), sex, imaging modality (radiograph, computed tomography [CT], or magnetic resonance imaging [MRI]), race (identified by the patients following the Institute of Medicine report on standardization of collection of race, ethnicity, and language data; ${ }^{27}$ categories included Asian, Black/African American, Native Hawaiian/ Pacific Islander, multiracial, Native American/Alaska Native, or white), ethnicity (Hispanic or not Hispanic), primary insurance at index (categorized as commercial, which included Medicare supplements; Veteran's Affairs (VA); self-pay; Medicare; or Medicaid); Charlson comorbidity index category (0, 
$1,2$, and $\geq 3) ;{ }^{28}$ receipt of prescriptions for opioids non-opioid pain medications pre-index, and the calendar time of the index image (categorized in 6month intervals following the intervention schedule from October 1, 2013-September 30, 2016). To determine each patient's SES, the study sites mapped patient addresses to Federal Information Processing System (FIPS) codes using geocoding software, which was then mapped to SES indexes derived from the 2010 Census Summary File 1 and the American Community Survey 2007 to 20115 -year estimate data $^{29}$ and categorized into quartiles. ${ }^{30}$ Using machine learning natural language processing, ${ }^{31}$ we extracted findings on the index image from the radiology text reports. These were categorized into 3 mutually exclusive groups: no findings, findings that were likely clinically unimportant (for example, disk bulge, disk space narrowing, annular fissure; hereafter termed "clinically unimportant findings"), and findings that were likely clinically important (hereafter termed "clinically important findings"; for example, moderate-severe spinal canal stenosis, nerve root compression, disk extrusion).

Because the providers who ordered the images may not have been the same as the providers who prescribed medications, we evaluated the proportions of patients who received opioid or non-opioid pain medication prescriptions from their imageordering providers. We also evaluated provider age, type (categorized into medical doctor [MD], doctor of osteopathic medicine [DO], or other [physician assistants and nurse practitioners; hereafter termed "PA/NP"]), sex, and specialty (family medicine, internal medicine, or other). Clinic characteristics included rural/urban, which we defined using the 2010 Rural-Urban Commuting Area (RUCA) codes, ${ }^{32}$ and clinic size (equal-size tertiles of small, medium, or large depending on the number of PCPs at the clinics).

\section{Outcomes}

To examine differences in the amount of prescribing, we subdivided patients who received any opioid or non-opioid pain prescriptions in the year following index into those with multiple periods with prescriptions versus only a single period with a prescription. Specifically, we divided the year after index into 4 quarters of 91 to 92 days and counted the number of quarters in which each patient received $\geq 1$ opioid or non-opioid pain medication prescription. We then created binary variables that were 0 if they received opioid or non-opioid pain medication prescriptions in 1 quarter and 1 if they received prescriptions in $>1$ quarter (hereafter termed "multiple prescriptions"). Thus, we had 4 binary outcome variables: (1) receipt of immediate prescriptions for opioids versus those who did not receive immediate opioid prescriptions (referent); (2) receipt of multiple prescriptions for opioids versus those who received only 1 opioid prescription (referent); (3) receipt of immediate prescriptions for non-opioid pain medications versus those who did not receive immediate non-opioid pain prescriptions (referent); and (4) receipt of multiple prescriptions for non-opioid pain prescriptions versus those who received only 1 non-opioid pain prescription (referent).

\section{Statistical Analysis}

We evaluated unadjusted relationships between the patient, provider, and clinic characteristics and those who did and did not receive prescriptions for opioid or (separately) non-opioid pain medications within 90 days after index ("immediate prescriptions"). We also examined the proportions of patients who received immediate prescriptions for (1) both opioid and non-opioid pain medication prescriptions, (2) only opioid prescriptions, (3) only non-opioid pain prescriptions, and (4) neither.

We used mixed models with random effects (which account for the fact that patients within PCPs and clinics would have been expected to have had prescribing patterns that were correlated) to estimate adjusted odds ratios (ORs) and 95\% confidence intervals (95\% CIs). We included all the patient-level, provider-level, and clinic-level variables in the same models. All models additionally adjusted for health care system and whether the image occurred during the control (no benchmark text present on the imaging report) or intervention (benchmark text present on the imaging report) period.

To evaluate whether concordance of patient and provider sex affected pain prescriptions, we conducted secondary, unadjusted analyses that examined the proportions of immediate and multiple pain medication prescriptions stratified by patient and provider sex. For these analyses, we only counted opioid or non-opioid pain prescriptions prescribed by the same provider who ordered the index images. 
Figure 1. Flow of patients in analyses stratified by whether patients received immediate opioid and/or non-opioid pain medication prescriptions. Abbreviation: LIRE, Lumbar Imaging with Reporting of Epidemiology.

$\mathrm{N}=238,886$
Total patients in LIRE
Study

Comparison 1: Immediate Vs No Immediate Opioid Prescriptions
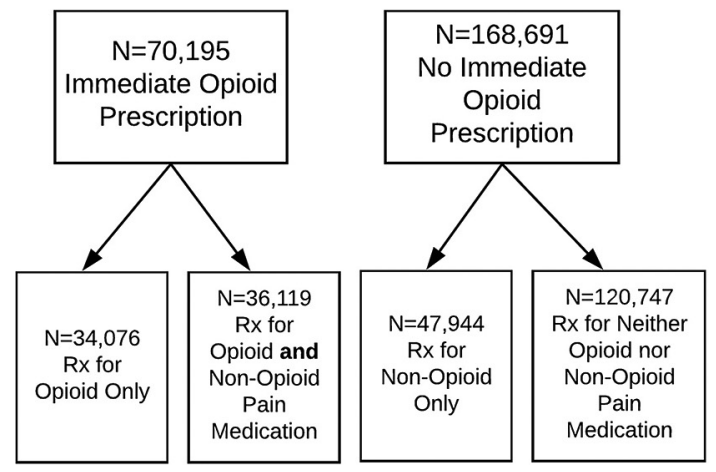

For patients who were missing values for race ( $n=28,932 ; 12.1 \%$ of the sample), we used the modes of the races in the zip codes where they received their index images to impute their races. ${ }^{33}$ We used the same methodology to impute SES for the $\mathrm{n}=6810$ (2.9\% of the population) patients missing SES. We conducted sensitivity analyses excluding the patients who were missing race or SES. A large proportion of patients were missing ethnicity data $(\mathrm{n}=163,514 ; 68 \%)$ but most $(99 \%)$ of these patients' data came from a health system that coded their patients' ethnicity as either "Hispanic" or "Unknown"; therefore, everyone whose ethnicity was recorded as "Unknown" was counted as not Hispanic for these analyses. Finally, patient primary insurance status was missing for a small proportion of our sample ( $\mathrm{n}=2697 ; 1.1 \%$ ); these patients were excluded from adjusted analyses. We used SAS software (version 9.4; Cary, North Carolina) for all analyses.

\section{Results}

A total of 238,886 patients were included in our analyses (Figure 1). Of these, 34,076 (14.3\%) received an immediate opioid prescription and no immediate non-opioid pain medication prescrip-

\section{Comparison 2: Immediate Vs No Immediate} Non-Opioid Pain Prescriptions
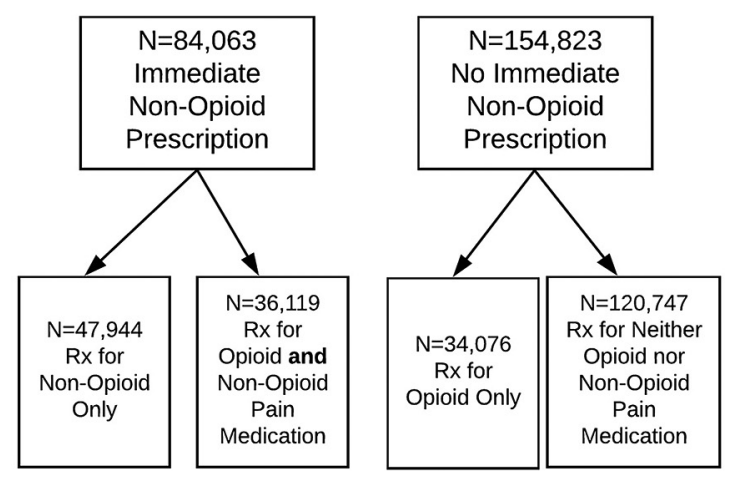

tion. A total of 47,944 (20.0\%) received only a nonopioid pain medication prescription and no immediate opioid prescription; 36,119 (15.1\%) received both immediate opioid and non-opioid pain medication prescriptions. Thus, approximately half of the cohort $(118,139 ; 49.5 \%)$ received at least 1 immediate pain medication prescription. A total of 61,259 (87\%) of patients who received immediate opioid prescriptions received $\geq 1$ immediate prescription from the same provider who ordered their index images, and $73,732(88 \%)$ of patients who received immediate non-opioid pain medication prescriptions received $\geq 1$ prescription from the image-ordering providers. Unadjusted patient-level, providerlevel, and clinic-level characteristics, stratified by whether the patients received immediate prescriptions for opioids or non-opioid pain medications, are shown in Table 1.

\section{Patient Race and Ethnicity}

Results of adjusted analyses are shown in Figures 2-5. Compared with whites, patients identified as Asian had reduced odds of receiving immediate opioid (OR, 0.53; 95\% CI, 0.51-0.56) or non-opioid (0.75; $0.73-0.77)$ pain medication prescriptions and of receiving multiple prescriptions (opioids: 0.60 ; $0.56-$ 
Table 1. Characteristics of Patients, Providers, and Clinics by Receipt of Opioid and Non-opioid Pain Medication Prescriptions within 90 Days After Index Image*

\begin{tabular}{|c|c|c|c|}
\hline & $\begin{array}{l}\text { All Patients n }(\%)^{\dagger} \\
\qquad \mathrm{N}=238,886\end{array}$ & $\begin{array}{l}\text { Received Immediate Opioid } \\
\text { Prescription n (\%) } \\
\mathrm{N}=70,195(29)\end{array}$ & $\begin{array}{c}\text { Received Immediate Non-opioid } \\
\text { Prescription } \mathrm{n}(\%)^{\ddagger} \\
\mathrm{N}=84,063(35)\end{array}$ \\
\hline \multicolumn{4}{|l|}{ Patient-Level Characteristics } \\
\hline \multicolumn{4}{|l|}{ Age group (years) } \\
\hline 18 to 39 & $43,342(18)$ & $9,679(22)$ & $15,695(36)$ \\
\hline 40 to 60 & $90,027(38)$ & $26,128(29)$ & $35,143(39)$ \\
\hline$>60$ & $105,517(44)$ & $34,388(33)$ & $33,225(31)$ \\
\hline \multicolumn{4}{|l|}{ Sex } \\
\hline Male & $101,499(42)$ & $28,956(29)$ & $33,365(33)$ \\
\hline Female & $137,387(58)$ & $41,239(30)$ & $50,498(37)$ \\
\hline \multicolumn{4}{|l|}{ Modality for index image } \\
\hline Radiograph & $192,435(81)$ & $55,466(29)$ & $70,234(37)$ \\
\hline Computed tomography & $943(0.4)$ & $412(44)$ & $288(31)$ \\
\hline Magnetic resonance imaging & $45,508(19)$ & $14,317(31)$ & $13,541(30)$ \\
\hline \multicolumn{4}{|l|}{ Race } \\
\hline Asian & $26,508(11)$ & $4,062(15)$ & $7,162(27)$ \\
\hline Black/African American & $23,642(10)$ & $7,678(33)$ & $8,416(36)$ \\
\hline Native Hawaiian/Pacific Islanders & $1,614(0.7)$ & $389(24)$ & $583(36)$ \\
\hline Native American/Alaska Native & $1,686(0.7)$ & $610(36)$ & $708(42)$ \\
\hline Multiracial & $1,005(0.4)$ & $296(29)$ & $376(37)$ \\
\hline White & $184,431(77)$ & $57,728(31)$ & $66,818(36)$ \\
\hline \multicolumn{4}{|l|}{ Ethnicity } \\
\hline Hispanic & $36,229(15)$ & $9,342(26)$ & $13,702(38)$ \\
\hline Not Hispanic & $202,657(85)$ & $60,853(30)$ & $70,361(35)$ \\
\hline \multicolumn{4}{|l|}{ Socioeconomic status quartile } \\
\hline 1 st & $15,824(7)$ & $5,446(34)$ & $6,333(40)$ \\
\hline 2nd & $31,213(13)$ & $10,574(34)$ & $12,215(39)$ \\
\hline $3 \mathrm{rd}$ & $57,564(24)$ & $18,808(33)$ & $21,879(38)$ \\
\hline 4th & $134,285(56)$ & $35,367(26)$ & $43,636(33)$ \\
\hline \multicolumn{4}{|l|}{ Insurance status } \\
\hline Medicare & $90,841(38)$ & $31,951(35)$ & $29,103(32)$ \\
\hline Medicaid/state-subsidized & $12,056(5)$ & $4,230(35)$ & $5,388(45)$ \\
\hline $\begin{array}{l}\text { Commercial (including Medicare } \\
\text { supplements) }\end{array}$ & $131,743(55)$ & $32,693(25)$ & $47,911(36)$ \\
\hline Veterans Administration & $248(0.1)$ & $60(24)$ & $84(34)$ \\
\hline Self-pay & $1,301(0.5)$ & $490(38)$ & $596(46)$ \\
\hline Unknown/missing & $2,697(1)$ & $771(29)$ & $981(36)$ \\
\hline \multicolumn{4}{|l|}{ Charlson comorbidity index } \\
\hline 0 & $153,079(64)$ & $38,227(25)$ & $53,108(35)$ \\
\hline 1 & $41,868(18)$ & $14,449(35)$ & $15,868(38)$ \\
\hline 2 & $23,211(10)$ & $8,214(35)$ & $7,828(34)$ \\
\hline $3+$ & $20,728(9)$ & $9,305(45)$ & $7,259(35)$ \\
\hline \multicolumn{4}{|c|}{$\begin{array}{l}\text { Prescription for opioid in } 120 \text { days before } \\
\text { index: }\end{array}$} \\
\hline Yes & $61,531(26)$ & $38,581(63)$ & $35,397(52)$ \\
\hline No & $177,355(74)$ & $31,614(18)$ & $48,666(29)$ \\
\hline \multicolumn{4}{|l|}{ Image finding status } \\
\hline None & $55,546(23)$ & $13,327(24)$ & $20,627(37)$ \\
\hline Clinically unimportant finding & $149,192(62)$ & $45,558(31)$ & $53,206(36)$ \\
\hline Clinically important finding & $34,148(14)$ & $11,310(33)$ & $10,230(30)$ \\
\hline
\end{tabular}




\begin{tabular}{|c|c|c|c|}
\hline & $\begin{array}{l}\text { All Patients n }(\%)^{\dagger} \\
\quad \mathrm{N}=238,886\end{array}$ & $\begin{array}{l}\text { Received Immediate Opioid } \\
\text { Prescription n (\%) } \\
\mathrm{N}=70,195(29)\end{array}$ & $\begin{array}{c}\text { Received Immediate Non-opioid } \\
\text { Prescription } \mathrm{n}(\%)^{\ddagger} \\
\mathrm{N}=84,063(35)\end{array}$ \\
\hline \multicolumn{4}{|l|}{ Time of index image } \\
\hline Oct 2013 to Mar 2014 & $48,233(20)$ & $16,087(33)$ & $16,909(35)$ \\
\hline Apr 2014 to Sept 2014 & $30,529(13)$ & $9,952(33)$ & $10,833(35)$ \\
\hline Oct 2014 to Mar 2015 & $39,424(17)$ & $11,730(30)$ & $14,204(36)$ \\
\hline Apr 2015 to Sept 2015 & $41,796(18)$ & $11,959(29)$ & $14,816(35)$ \\
\hline Oct 2015 to Mar 2016 & $39,177(16)$ & $10,526(27)$ & $13,726(35)$ \\
\hline Apr 2016 to Sept 2016 & $39,727(17)$ & $9,941(25)$ & $13,575(34)$ \\
\hline \multicolumn{4}{|l|}{$\begin{array}{l}\text { Image Ordering Provider-Level } \\
\text { Characteristics }\end{array}$} \\
\hline Provider mean age (IQR) & $49.3(43$ to 55$)$ & $49.6(43$ to 56$)$ & $49.0(43$ to 55$)$ \\
\hline \multicolumn{4}{|l|}{ Provider type } \\
\hline Doctor Osteopathy & $17,288(7)$ & $5,167(30)$ & $6,542(38)$ \\
\hline Medical Doctor & $213,524(89)$ & $62,250(29)$ & $74,170(35)$ \\
\hline Physician Assistant/Nurse Practitioner & $8,074(3)$ & $2,778(34)$ & $3,351(42)$ \\
\hline \multicolumn{4}{|l|}{ Provider sex } \\
\hline Female & $125,520(53)$ & $35,315(28)$ & $44,206(35)$ \\
\hline Male & $113,366(47)$ & $34,880(31)$ & $39,857(35)$ \\
\hline \multicolumn{4}{|l|}{ Provider specialty } \\
\hline Family medicine & $117,072(49)$ & $36,042(31)$ & $44,100(38)$ \\
\hline Internal medicine & $119,842(50)$ & $33,602(28)$ & $39,278(33)$ \\
\hline Other & $1972(0.8)$ & $551(28)$ & $685(35)$ \\
\hline \multicolumn{4}{|l|}{ Imaging Clinic-Level Characteristics } \\
\hline \multicolumn{4}{|l|}{ Rural-urban commuting area type } \\
\hline Urban & $231,352(97)$ & $67,391(29)$ & $81,184(35)$ \\
\hline Large rural & $4,106(1.7)$ & $1,436(35)$ & $1,569(38)$ \\
\hline Small rural & $3,043(1.3)$ & $1,247(41)$ & $1,215(40)$ \\
\hline Isolated small rural & $385(0.2)$ & $121(31)$ & $95(25)$ \\
\hline \multicolumn{4}{|l|}{ Clinic size } \\
\hline Small & $48,053(20)$ & $14,044(29)$ & $15,725(33)$ \\
\hline Medium & $74,035(31)$ & $22,315(30)$ & $25,014(34)$ \\
\hline Large & $116,798(49)$ & $33,836(29)$ & $43,324(37)$ \\
\hline
\end{tabular}

IQR, Interquartile range.

*Note that 36,119 patients ( $15 \%$ of the total) received prescriptions for both opioid and non-opioid pain medications within 90 days and are therefore represented in multiple columns; similarly, $\mathrm{n}=120,747$ received neither immediate opioid nor non-opioid pain prescriptions.

${ }^{\dagger}$ Percentages presented in columns.

${ }^{\ddagger}$ Percentages presented in rows.

0.64; non-opioids: $0.69 ; 0.66-0.73)$. Patients identified as Black $(0.92 ; 0.89-0.96)$, Native Hawaiian/ Pacific Islander (0.73; $0.64-0.83)$, or multiracial (0.84; 0.71-0.98) also had reduced odds of receiving immediate opioid prescriptions, but we did not observe statistically significant associations with the receipt of multiple opioid prescriptions or immediate non-opioid pain prescriptions. However, patients identified as Black $(0.87 ; 0.83-0.91)$ or Native Hawaiian $(0.83 ; 0.71-0.97)$ had reduced odds relative to whites for receiving multiple prescriptions for non-opioid pain medications. Patients identified as Native American/Alaskan had greater odds of receiving immediate non-opioid pain medications than whites $(1.12 ; 1.01-1.24)$. Patients identified as Hispanic had reduced odds of receiving immediate prescriptions for opioids $(0.79 ; 0.770 .82)$ and of receiving multiple opioid $(0.80 ; 0.77-0.84)$ or nonopioid pain medications $(0.86 ; 0.83-0.89)$ within a year, but they had greater odds of receiving immediate non-opioid pain medications prescriptions (1.02; $1.00-1.05)$. 
Figure 2. Adjusted odds ratios (ORs) and 95\% confidence intervals (95\% CIs) of patient-level (patient age, sex, index imaging modality, race, ethnicity, socioeconomic status, insurance status, Charlson comorbidity index, prescriptions for opioids in the $\mathbf{1 2 0}$ days prior to index, image finding status, and time of index image), providerlevel (provider age, provider type, provider sex, and provider specialty), and clinic-level (rural urban commuting area type and clinic size) variables associated with receipt of immediate prescriptions for opioids within 90 days of receipt of lower back imaging. Abbreviations: CT, computed tomography; DO, doctor of osteopathic medicine; MD, medical doctor; MRI, magnetic resonance imaging; SES, socioeconomic status; VA, Veteran's Affairs.

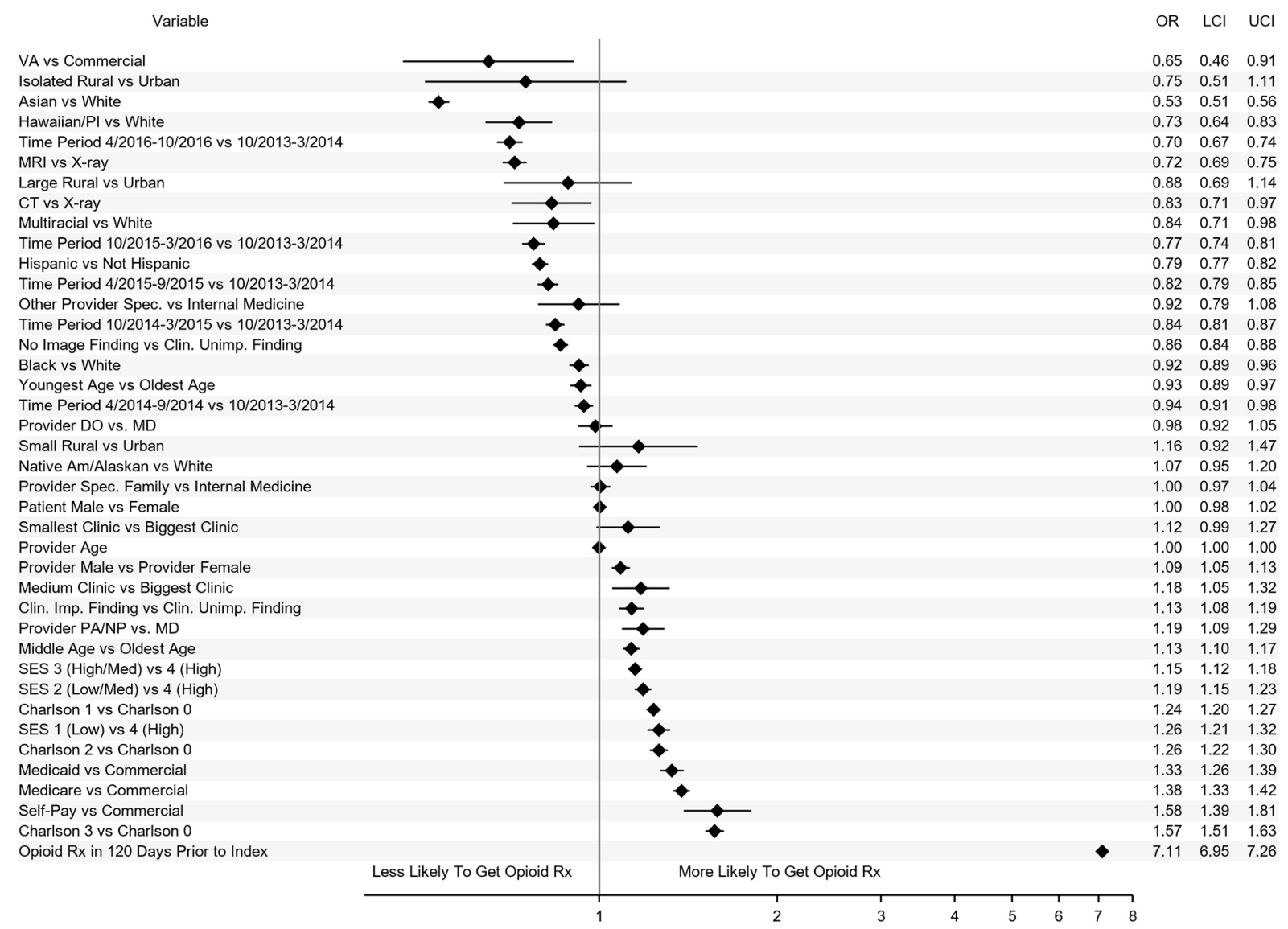

\section{Index Imaging}

Compared with patients with clinically unimportant findings on their index images, patients with no findings had reduced odds of receiving immediate opioid $(0.86 ; 0.84-0.88)$ or non-opioid (0.96; 0.94-0.99) prescriptions or of receiving multiple prescriptions (opioids: 0.87; 0.84-0.91; non-opioids: $0.92 ; 0.89-0.95)$. Patients with clinically important findings had reduced odds of receiving multiple opioid prescriptions compared with patients with clinically unimportant findings $(0.91 ; 0.84-0.98)$.

\section{Other Patient-Level Factors}

Patients insured by the VA were less likely to have received prescriptions for immediate opioids $(0.65$;
$0.46-0.91)$ or non-opioid pain medications $(0.73$; $0.55-0.98)$ than those with commercial insurance. Patients who received pre-index prescriptions for opioid non-opioid pain medications had increased odds of receiving immediate opioid or non-opioid pain prescriptions and of receiving multiple prescriptions after index; these ORs ranged from 2.75 (95\% CI, 2.70-2.80) for an immediate non-opioid pain prescription to 7.11 (95\% CI, 6.95-7.26) for immediate opioid prescriptions; ORs for all other characteristics did not exceed 1.60. Patients in the youngest age category had reduced odds of receiving immediate opioid prescriptions $(0.93 ; 0.89$ $0.97)$ and multiple prescriptions for non-opioids $(0.78 ; 0.74-0.82)$ but increased odds $(1.13 ; 1.09$ 1.17) of receiving immediate non-opioid pain 
Figure 3. Adjusted odds ratios (ORs) and 95\% confidence intervals (95\% CIs) of patient-level (patient age, sex, index imaging modality, race, ethnicity, socioeconomic status, insurance status, Charlson comorbidity index, prescriptions for opioids in the 120 days prior to index, image finding status, and time of index image), provider-level (provider age, provider type, provider sex, and provider specialty), and clinic-level (rural urban commuting area type and clinic size) variables associated with having 1 quarter (referent category) versus multiple quarters with at least 1 opioid prescription within a year of index low back image. Abbreviations: DO, doctor of osteopathic medicine; MD, medical doctor; PA/NP, physician assistants and nurse practitioners; SES, socioeconomic status.

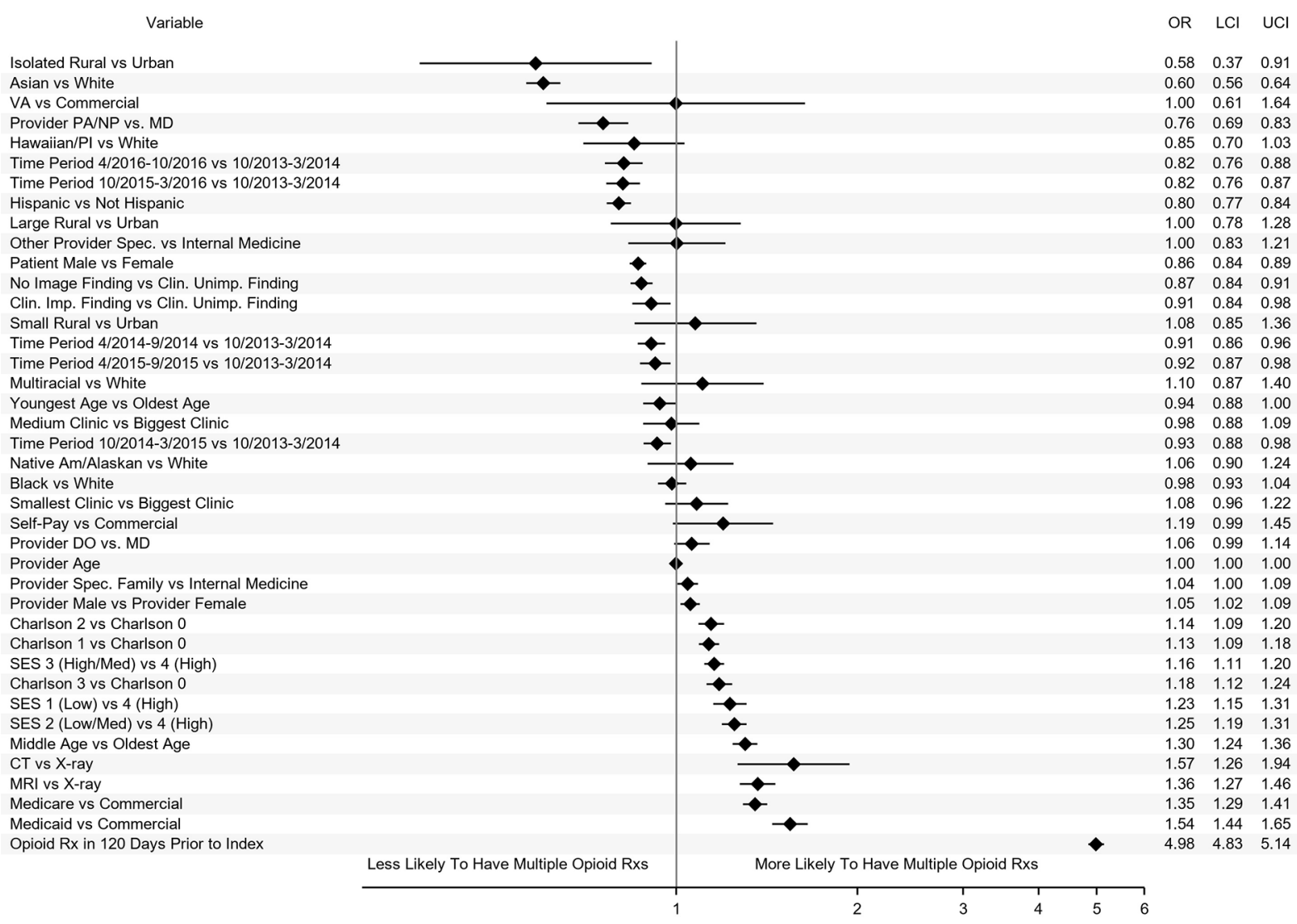

prescriptions compared with patients in the oldest age-group. Patients whose index images occurred later in calendar time had reduced odds of receiving prescription pain medications by almost all metrics than patients who received their images earlier.

\section{Patient and Provider Sex}

Relative to female patients, males had reduced odds of receiving immediate prescriptions for nonopioid pain medications $(0.90 ; 0.89-0.92)$ or multiple prescriptions for opioids $(0.86 ; 0.84-0.89)$ or non-opioids $(0.76 ; 0.74-0.78)$. However, patients whose index-image ordering providers were male had increased odds of receiving immediate (1.09; $1.05-1.13)$ and multiple prescriptions for opioids $(1.05 ; 1.02-1.09)$ or non-opioids (1.06; 1.03-1.09). Though male and female patients of female providers received opioid and non-opioid pain medication prescriptions equally, 29\% of female patients of male providers received immediate opioid prescriptions compared with only $26 \%$ of male patients of male providers (Supplemental Table 2). Similarly, 33\% of female patients of male providers received immediate prescriptions for non-opioid pain medications in contrast to $29 \%$ of male patients of male providers. Higher proportions of female patients of both male and female providers received multiple prescriptions for opioids and non-opioid pain medications compared with male patients.

\section{Clinic-Level Factors}

Patients who received their index images at medium-sized relative to large clinics had increased 
Figure 4. Adjusted odds ratios (ORs) and 95\% confidence intervals (95\% CIs) of patient-level (patient age, sex, index imaging modality, race, ethnicity, socioeconomic status, insurance status, Charlson comorbidity index, prescriptions for non-opioid pain medications in the $\mathbf{1 2 0}$ days prior to index, image finding status, and time of index image), provider-level (provider age, provider type, provider sex, and provider specialty), and clinic-level (rural urban commuting area type and clinic size) variables associated with receipt of immediate prescriptions for non-opioid pain medications within 90 days of receipt of lower back imaging. Abbreviations: CT, computed tomography; DO, doctor of osteopathic medicine; MD, medical doctor; PANP, physician assistants and nurse practitioners; SES, socioeconomic status; VA, Veteran's Affairs.

Variable

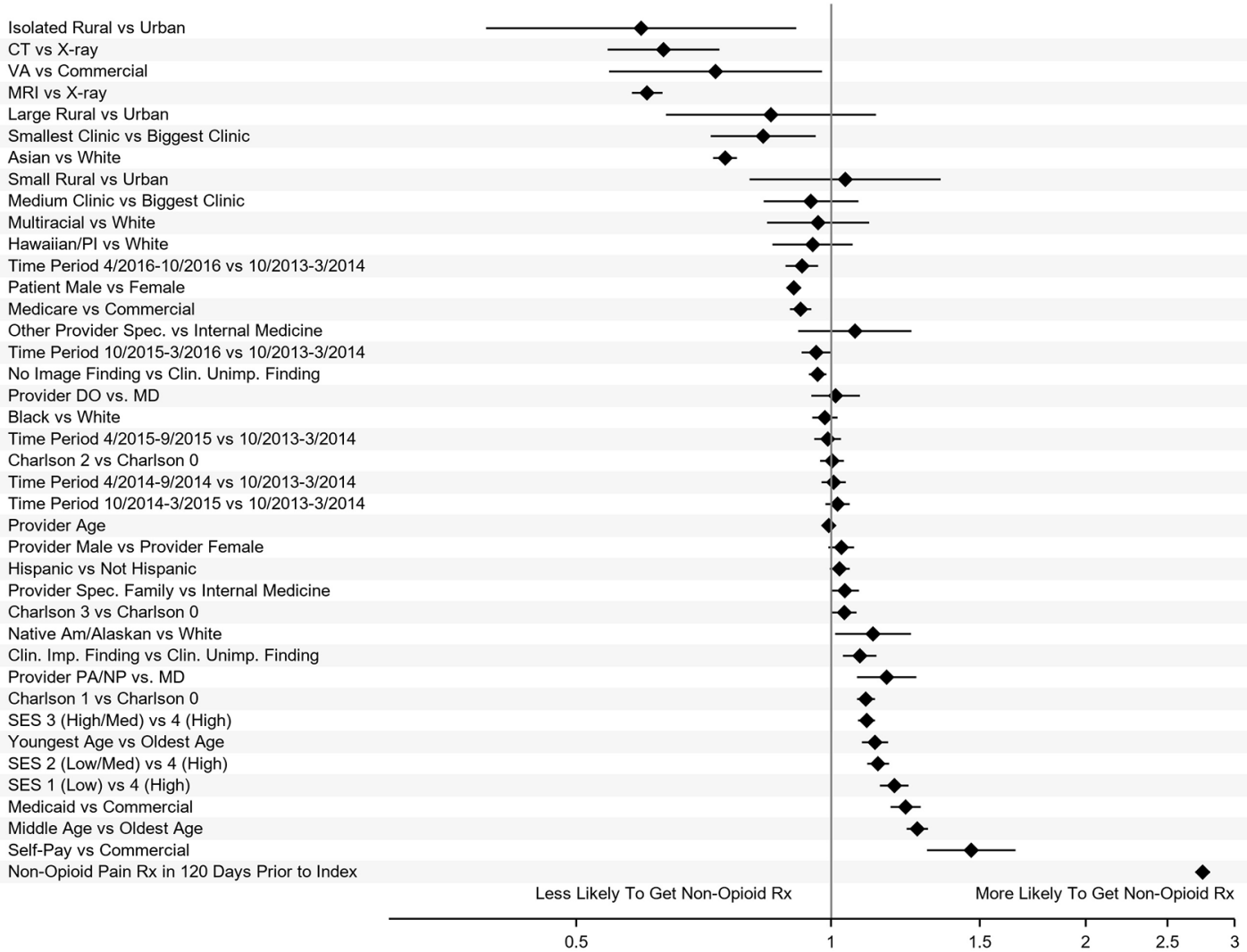

$\begin{array}{lll}0.60 & 0.39 & 0.91\end{array}$ $\begin{array}{lll}0.63 & 0.54 & 0.74\end{array}$ $\begin{array}{lll}0.73 & 0.55 & 0.98\end{array}$ $\begin{array}{lll}0.61 & 0.58 & 0.63\end{array}$ $\begin{array}{lll}0.85 & 0.64 & 1.13\end{array}$ $\begin{array}{lll}0.83 & 0.72 & 0.96\end{array}$ $\begin{array}{lll}0.75 & 0.73 & 0.77\end{array}$ $\begin{array}{lll}1.04 & 0.80 & 1.35\end{array}$ $\begin{array}{lll}0.95 & 0.83 & 1.08\end{array}$ $\begin{array}{lll}0.97 & 0.84 & 1.11\end{array}$ $\begin{array}{lll}0.95 & 0.85 & 1.06\end{array}$ $\begin{array}{lll}0.92 & 0.88 & 0.97\end{array}$ $\begin{array}{lll}0.90 & 0.89 & 0.92\end{array}$ $\begin{array}{llll}0.92 & 0.89 & 0.95\end{array}$ $\begin{array}{llll}0.92 & 0.89 & 0.95 \\ 1.07 & 0.91 & 1.24\end{array}$ $\begin{array}{lll}0.96 & 0.92 & 1.00\end{array}$ $\begin{array}{lll}0.96 & 0.94 & 0.99\end{array}$ $\begin{array}{lll}1.01 & 0.95 & 1.08\end{array}$ $\begin{array}{lll}0.98 & 0.95 & 1.02\end{array}$ $\begin{array}{lll}0.99 & 0.95 & 1.03\end{array}$ $\begin{array}{lll}1.00 & 0.97 & 1.03\end{array}$ $\begin{array}{lll}1.01 & 0.97 & 1.04\end{array}$ $\begin{array}{lll}1.02 & 0.99 & 1.05\end{array}$ $\begin{array}{lll}0.99 & 0.99 & 1.00\end{array}$ $\begin{array}{lll}1.03 & 0.99 & 1.06\end{array}$ $1.02 \quad 1.00 \quad 1.05$ $\begin{array}{lll}1.02 & 1.00 & 1.05\end{array}$ $1.04 \quad 1.00 \quad 1.07$ $\begin{array}{lll}1.04 & 1.00 & 1.07 \\ 1.12 & 1.01 & 1.24\end{array}$ $\begin{array}{lll}1.08 & 1.03 & 1.13\end{array}$ $\begin{array}{lll}1.16 & 1.07 & 1.26\end{array}$ $\begin{array}{lll}1.10 & 1.07 & 1.13\end{array}$ $\begin{array}{lll}1.10 & 1.08 & 1.13\end{array}$ $\begin{array}{lll}1.13 & 1.09 & 1.17\end{array}$ $\begin{array}{lll}1.14 & 1.10 & 1.17\end{array}$ $\begin{array}{lll}1.19 & 1.14 & 1.23\end{array}$

odds of receiving immediate opioid prescriptions $(1.18 ; 1.05-1.32)$. Patients whose index images took place at the smallest clinics had reduced odds of receiving immediate non-opioid pain prescriptions (0.83; 0.72-0.96). Patients whose index images occurred at isolated rural relative to urban clinics had reduced odds of receiving immediate nonopioid pain prescriptions $(0.60 ; 0.39-0.91)$ and of receiving multiple opioids $(0.58 ; 0.37-0.91)$ or non-opioid $(0.60 ; 0.40-0.90)$ pain prescriptions.

Sensitivity analyses that excluded patients who were missing race or SES revealed very similar results to those described above (Supplemental Figures 1 to 4). For example, in the main analysis in which race was imputed, patients identified as Black had $8 \%$ reduced odds ( $95 \%$ CI, $4 \%$ to $11 \%$ ) compared with whites of receiving immediate prescriptions for opioids; in the sensitivity analysis in which those with missing race and SES were excluded, Blacks had 10\% reduced odds (95\% CI, $6 \%$ to $13 \%$ ) of receiving immediate opioid prescriptions compared with whites.

\section{Discussion}

We report patient-level, provider-level, and cliniclevel characteristics associated with pain medication prescribing patterns that have not been welldescribed in past literature. Notably, we found that Asian patients had lower odds of receiving opioid or non-opioid pain medication prescriptions than white patients across all measures of those 
Figure 5. Adjusted odds ratios (ORs) and 95\% confidence intervals (95\% CIs) of patient-level (patient age, sex, index imaging modality, race, ethnicity, socioeconomic status, insurance status, Charlson comorbidity index, prescriptions for non-opioid pain medications in the $\mathbf{1 2 0}$ days prior to index, image finding status, and time of index image), provider-level (provider age, provider type, provider sex, and provider specialty), and clinic-level (rural urban commuting area type and clinic size) variables associated with having 1 quarter (referent category) versus multiple quarters with at least 1 non-opioid pain medication prescription within 1 year of index low back image. Abbreviations: CT, computed tomography; DO, doctor of osteopathic medicine; MD, medical doctor; PA/ NP, physician assistants and nurse practitioners; SES, socioeconomic status; VA, Veteran's Affairs.

\section{Variable}

Isolated Rural vs Urban

Time Period 4/2016-10/2016 vs 10/2013-3/2014

Asian vs White

VA vs Commercial

Hawaiian/PI vs White

Patient Male vs Female

Youngest Age vs Oldest Age

Time Period 10/2015-3/2016 vs 10/2013-3/2014

Large Rural vs Urban

Small Rural vs Urban

Provider PA/NP vs. MD

Multiracial vs White

Hispanic vs Not Hispanic

Black vs White

Time Period 4/2015-9/2015 vs 10/2013-3/2014

CT vs X-ray

No Image Finding vs Clin. Unimp. Finding

Medium Clinic vs Biggest Clinic

Self-Pay vs Commercial

Smallest Clinic vs Biggest Clinic

Time Period 4/2014-9/2014 vs 10/2013-3/2014

Provider DO vs. MD

Clin. Imp. Finding vs Clin. Unimp. Finding

Time Period 10/2014-3/2015 vs 10/2013-3/2014

Provider Age

Provider Spec. Family vs Internal Medicine

Other Provider Spec. vs Internal Medicine

Native Am/Alaskan vs White

SES 1 (Low) vs 4 (High)

Provider Male vs Provider Female

SES 3 (High/Med) vs 4 (High)

SES 2 (Low/Med) vs 4 (High)

Middle Age vs Oldest Age

MRI vs X-ray

Charlson 2 vs Charlson 0

Charlson 1 vs Charlson 0

Charlson 3 vs Charlson 0

Medicare vs Commercia

Medicaid vs Commercia

Non-Opioid Pain Rx in 120 Days Prior to Index

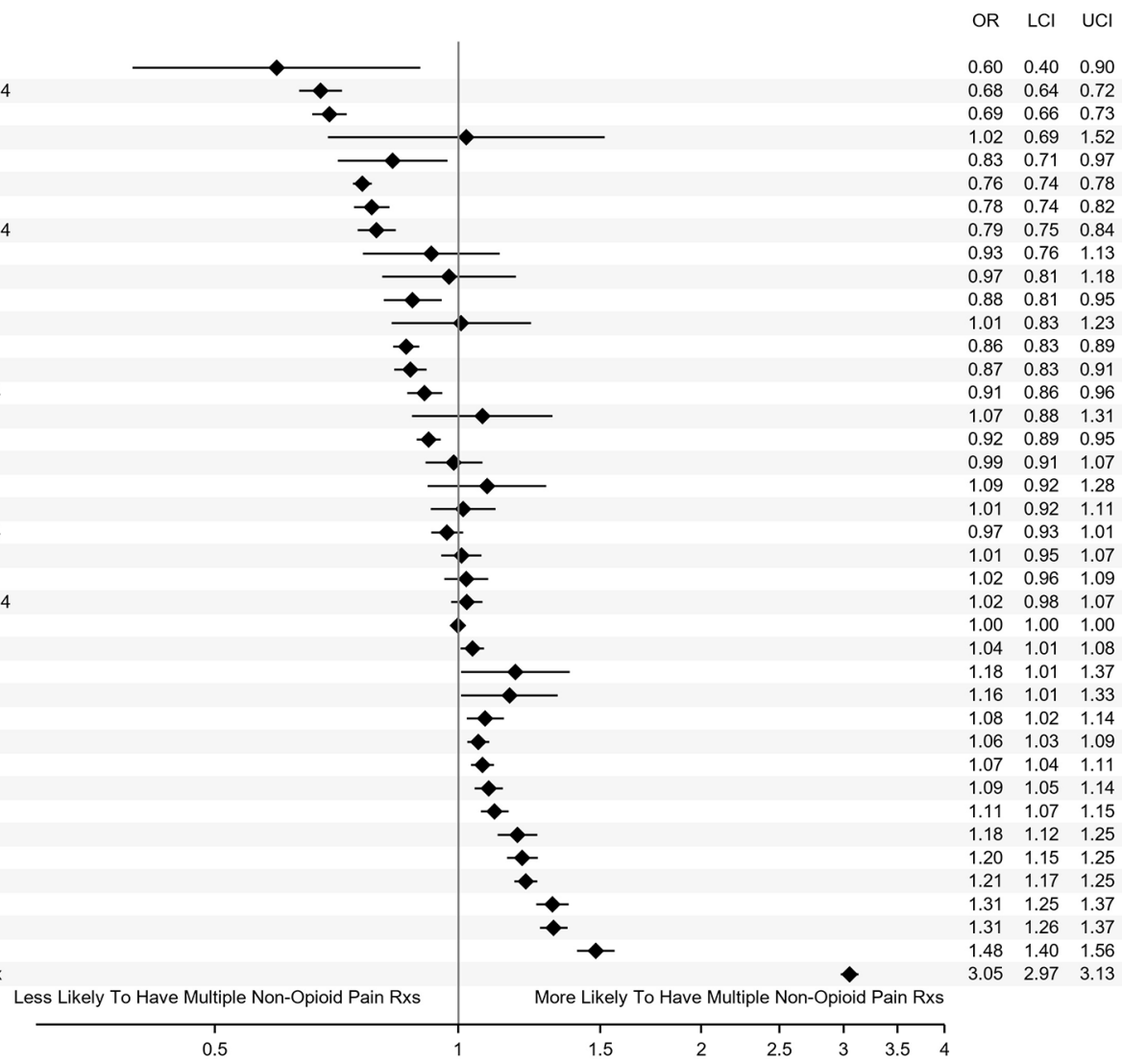

prescriptions. We also found that, compared with older patients, middle-aged patients had higher adjusted odds of receiving pain medication prescriptions in all outcome measures. Patients who had prescriptions before index for opioid or nonopioid pain medications also had greater odds of receiving prescriptions for pain medications postindex. In addition, consistent with prior research, we found that patients identified as Black/African American, ${ }^{12,34}$ identified as Hispanic, ${ }^{12,34}$ of higher SES, ${ }^{14}$ with no comorbidities, ${ }^{35}$ or who received low back imaging in more recent years ${ }^{7,8}$ had reduced odds of receiving immediate prescriptions for opioids. Likely, the reduction in opioid prescriptions in the later years of our study was influenced both by the increasing proportion of patients whose images contained the benchmark text for the LIRE study, which was associated with small but significant decreases in the likelihood of subsequent opioid prescriptions, ${ }^{23}$ as well as by increasingly broad societal concern about the harms associated with opioid prescriptions. These concerns were summarized in the 2016 Centers for Disease Control and Prevention (CDC) opioid prescribing guidelines, which called attention to the lack of evidence supporting the efficacy of long-term opioid therapy and its potential harms.

Our sample was notable for having substantial numbers of patients in racial categories for whom trends in pain medication prescribing have not been well-described, including patients identified as Asian, Native Hawaiian, Native American, or multiracial. 
Rates of opioid prescribing tend to be dramatically lower in Asian countries than in the US, likely due to variances in cultural norms and legal regulations. ${ }^{36,37}$ It is possible that reduced expectations of pain prescriptions were also present in the populations of Asian patients in this study, which resulted in their receiving fewer pain prescriptions than whites. We did not have data on patient preferences or attitudes to assess this hypothesis, but future work should examine this in greater detail.

We did not observe significant differences in the opioid outcomes among patients identified as Native American/Alaskan Native versus whites, but they had greater odds of receiving immediate prescriptions for non-opioids. A recent study found that fatal opioid-involved overdoses, which included both prescription and illegal drugs, in American Indian/Alaska Natives had increased from a rate similar to that of whites in 1999 to 2001 to 4.1 times greater than that in whites by 2013 to $2015 .^{38}$ Given this trend, it is possible that providers were prescribing non-opioid pain medications in this population to reduce their exposure to opioids, but we could not address this hypothesis with our data.

Our finding that patients who received pre-index prescriptions for pain medications had much higher odds of receiving subsequent pain prescriptions may reflect several factors. Providers may be subject to "prescribing inertia" and continue patients on medications they are already on, regardless of their efficacy. ${ }^{39}$ In addition, patients who received previous prescriptions for pain medications may have been more likely to request pain prescriptions following their low back imaging, particularly if they were experiencing acute episodes of low back pain. We were unable to evaluate the appropriateness of the pain medication prescriptions.

Although some prior research has reported that male patients are less likely to be prescribed opioids than female patients, ${ }^{16-18}$ we found no associations between patient sex and receipt of immediate opioid prescriptions, but females had greater odds of having multiple pain prescriptions. We found that male image-ordering providers were more likely to write immediate opioid prescriptions and write multiple prescriptions for opioids or non-opioid pain medications than female providers. This finding is the opposite of the conclusions of an analysis of PCPs in the United Kingdom (not specific to patients with low back pain), which found that female providers were more likely to prescribe opioids. ${ }^{40}$ In unadjusted analyses, we also found that male providers were slightly more likely to prescribe pain medications to female patients than males, and higher proportions of female patients received multiple prescriptions from both female and male providers. Concordance of patient-provider sex and pain medication prescribing has not been widely researched, but a study of prescribing patterns in an emergency department found that male physicians were more likely to prescribe opioids to male patients and female physicians were more likely to prescribe to female patients. ${ }^{41}$ Further investigation into these patterns that can account for potentially confounding factors is necessary to replicate and to understand the reasons for these findings.

\section{Limitations}

Our study included a large and diverse cohort of patients, but it has some limitations. First, although all patients had received low back imaging, we cannot be certain that the index image was ordered because of low back pain. We also lacked information on the duration of pain, if any, and thus could not examine prescribing differences for patients with acute versus chronic pain. We could not be sure that prescriptions were for low back pain rather than some other indication, including opioid use disorder. Second, we lacked data for over-the-counter medications. Third, patient race and ethnicity may have been misclassified. Although the policy at each of the health systems in our study was to have patients selfidentify, we could not be certain that race and ethnicity in the EMR were identified by patients themselves or by staff. However, some genomics literature suggests that observer-assigned race is concordant with ancestry. ${ }^{42,43}$ Imputing missing race using the most common races in the zip code where the imaging took place may have misclassified some patients into incorrect racial categories. Although we found similar results when we conducted sensitivity analyses that excluded patients with missing data, our results may have been subject to bias. Fourth, data were not available on confounders that may have affected opioid prescribing, including education, employment status, smoking status, anxiety and depression, pain severity, and use of health-related services (eg, acupuncture) that were not available in the EMR. While ICD-9-CM and ICD-10-CM diagnosis codes for opioid use disorder (OUD) exist, they have been shown to be 
underutilized in EMR, ${ }^{44}$ and we found very few instances of patients with these codes in our data, so we were unable to adjust for OUD. Similarly, we lacked data on provider characteristics that might affect pain medication prescribing, such as race, cultural and pain management competencies, and language capabilities. Finally, given the number of characteristics that we analyzed, some of the associations found could have been due to chance.

\section{Conclusion}

We highlighted several novel patient-level characteristics associated with the receipt of prescription pain medications, including fewer immediate pain medication prescriptions among patients identified as Asian, Black, Native Hawaiian/Pacific Islander, or multiracial and greater immediate prescribing of non-opioid pain medications for patients identified as Native American/Alaska Native. Further large, populationbased studies are needed to replicate and better understand these findings. Patients who had prior prescriptions for opioid or non-opioid pain medications were more likely to receive subsequent prescriptions for pain medications. These findings could be useful in future studies to investigate how health care systems could identify opportunities to optimize pain medication management for individual patients.

To see this article online, please go to: http://jabfm.org/content/ 34/5/950.full.

\section{References}

1. Urits I, Burshtein A, Sharma M, et al. Low back pain, a comprehensive review: pathophysiology, diagnosis, and treatment. Curr Pain Headache Rep 2019;23:23.

2. Chou R, Qaseem A, Snow V, et al. American Pain Society Low Back Pain Guidelines Panel. Diagnosis and treatment of low back pain: a joint clinical practice guideline from the American College of Physicians and the American Pain Society. Intern Med Ann 2007;147:478-491.

3. North American Spine Society. Choosing Wisely. Available from: https://www.choosingwisely.org/ clinician-lists/nass-opioids-for-acute-or-chronic-lowback-pain/. Published 2019. Accessed April 27, 2020.

4. Qaseem A, Wilt TJ, McLean RM, Forciea MA. Clinical guidelines committee of the American College of Physicians noninvasive treatments for acute, subacute, and chronic low back pain: a clinical practice guideline from the American College of Physicians. Noninvasive treatments for acute, subacute, and chronic low back pain: a clinical practice guideline from the American College of Physicians. Ann Intern Med 2017;166:514-530.

5. National Institute for Health Care and Excellence (NICE). Low Back Pain and Sciatica in Over 16s: Assessment and Management. In: Low Back Pain and Sciatica in Over 16s: Assessment and Management. London 2016.

6. van Tulder M, Becker A, Bekkering T, et al. COST B13 Working Group on Guidelines for the Management of Acute Low Back Pain in Primary Care. Chapter 3. European guidelines for the management of acute nonspecific low back pain in primary care. Eur Spine J 2006;15 Suppl 2:S169-191.

7. Hoots BE, Xu L, Kariisa M, et al. 2018 Annual surveillance report of drug-related risks and outcomes-United States. Published 2018.

8. Raad M, Pakpoor J, Harris AB, et al. Opioid prescriptions for new low back pain: trends and variability by state. J Am Board Fam Med 2020;33:138-142.

9. Dowell D, Haegerich TM, Chou R. CDC guideline for prescribing opioids for chronic pain-United States, 2016. JAMA 2016;315:1624.

10. Lee P, Le Saux M, Siegel R, et al. Racial and ethnic disparities in the management of acute pain in US emergency departments: meta-analysis and systematic review. Am J Emerg Med 2019;37:1770-1777.

11. Milani CJ, Rundell SD, Jarvik JG, et al. Associations of race and ethnicity with patientreported outcomes and health care utilization among older adults initiating a new episode of care for back pain. Spine 2018;43:1007-1017.

12. Meghani SH, Byun E, Gallagher RM. Time to take stock: a meta-analysis and systematic review of analgesic treatment disparities for pain in the United States. Pain Med 2012;13:150-174.

13. Harrison JM, Lagisetty P, Sites BD, Guo C, Davis MA. Trends in prescription pain medication use by race/ethnicity among US adults with noncancer pain, 2000-2015. Am J Public Health 2018;108:788-790.

14. Gebauer S, Salas J, Scherrer JF. Neighborhood socioeconomic status and receipt of opioid medication for new back pain diagnosis. J Am Board Fam Med 2017;30:775-783.

15. Jeffrey Kao MC, Minh LC, Huang GY, Mitra R, Smuck M. Trends in ambulatory physician opioid prescription in the United States, 1997-2009. PM R 2014;6:575-582.e574.

16. Smith JA, Fuino RL, Pesis-Katz I, et al. Differences in opioid prescribing in low back pain patients with and without depression: a cross-sectional study of a national sample from the United States. Pain Rep 2017;2:e606.

17. Serdarevic M, Striley CW, Cottler LB. Sex differences in prescription opioid use. Curr Opin Psychiatry 2017;30:238-246.

18. Back SE, Payne RL, Simpson AN, Brady KT. Gender and prescription opioids: findings from the 
National Survey on Drug Use and Health. Addict Behav 2010;35:1001-1007.

19. Agnoli A, Jerant A, Franks P. Prescription opioids and patient sex: a national cross-sectional study. J Womens Health (Larchmt) in press. 2020.

20. Luu H, Slavova S, Freeman PR, Lofwall M, Browning S, Bush H. Trends and patterns of opioid analgesic prescribing: regional and rural-urban variations in Kentucky from 2012 to 2015. J Rural Health 2019;35:97-107.

21. Rigg KK, Monnat SM, Chavez MN. Opioid-related mortality in rural America: Geographic heterogeneity and intervention strategies. Int J Drug Policy 2018;57:119-129.

22. Ganem VJ, Mora AG, Varney SM, Bebarta VS. Emergency department opioid prescribing practices for chronic pain: a 3-year analysis. J Med Toxicol 2015;11:288-294.

23. Jarvik JG, Meier EN, James KT, et al. The effect of including benchmark prevalence data of common imaging findings in spine image reports on health care utilization among adults undergoing spine imaging: a stepped-wedge randomized clinical trial. JAMA Netw Open 2020;3:e2015713.

24. Jarvik JG, Comstock BA, James KT, et al. Lumbar Imaging With Reporting Of Epidemiology (LIRE)-Protocol for a pragmatic cluster randomized trial. Contemp Clin Trials 2015;45:157-63.

25. Brinjikji W, Luetmer PH, Comstock B, et al. Systematic literature review of imaging features of spinal degeneration in asymptomatic populations. AJNR Am J Neuroradiol 2015;36:811-816.

26. Roland M, van Tulder M. Should radiologists change the way they report plain radiography of the spine? Lancet 1998;352:229-230.

27. Nerenz DR, McFadden B, Ulmer C. Race, ethnicity, and language data: standardization for health care quality improvement. Published 2009.

28. Quan H, Li B, Couris CM, et al. Updating and validating the Charlson comorbidity index and score for risk adjustment in hospital discharge abstracts using data from 6 countries. Am J Epidemiol 2011;173:676-82.

29. Anderson ML. Linking Demographic and Socioeconomic Data to the Electronic Health Record. Living Textbook of Pragmatic Clinical Trials. Available from: https://rethinkingclinicaltrials.org/resources/linkingelectronic-health-record-data-to-socioeconomic-statusmethods-and-documentation/. Published 2014. Accessed August 7, 2020.

30. Agency for Healthcare Research and Quality. Chapter 3: Creation of New Race-Ethnicity Codes and SES Indicators for Medicare Beneficiaries. Available from: https://archive.ahrq.gov/research/findings/final-reports/ medicareindicators/medicareindicators $3 . \mathrm{html}$. Published 2008. Updated 2008. Accessed May 23, 2018.

31. Tan WK, Hassanpour S, Heagerty PJ, et al. Comparison of natural language processing rules- based and machine-learning systems to identify lumbar spine imaging findings related to low back pain. Acad Radiol 2018;25:1422-1432.

32. United States Department of Agriculture. Documentation 2010 Rural-Urban Commuting Area (RUCA) Codes. Available from: https://www. ers.usda.gov/data-products/rural-urban-commutingarea-codes/documentation/. Published 2019. Accessed March 11, 2020.

33. Xu X, Chong W, Li S, Arabo A, Xiao J. MIAEC: Missing data imputation based on the evidence chain. IEEE Access 2018;6:12983-12992.

34. Ly DP. Racial and ethnic disparities in the evaluation and management of pain in the outpatient setting, 2006-2015. Pain Med 2019;20:223-232.

35. Dobscha SK, Morasco BJ, Duckart JP, Macey T, Deyo RA. Correlates of prescription opioid initiation and long-term opioid use in veterans with persistent pain. Clin J Pain 2013;29:102-108.

36. Onishi E, Kobayashi T, Dexter E, Marino M, Maeno T, Deyo RA. Comparison of opioid prescribing patterns in the United States and Japan: primary care physicians' attitudes and perceptions. J Am Board Fam Med 2017;30:248-254.

37. Deyo RA, Von Korff M, Duhrkoop D. Opioids for low back pain. BMJ 2015;350:g6380.

38. Joshi S, Weiser T, Warren-Mears V. Drug, opioidinvolved, and heroin-involved overdose deaths among American Indians and Alaska Natives Washington, 1999-2015. MMWR Morb Mortal Wkly Rep 2018;67:1384-1387.

39. Steinman MA, Landefeld CS. Overcoming inertia to improve medication use and deprescribing. JAMA 2018;320:1867-1869.

40. Chen TC, Chen LC, Kerry M, Knaggs RD. Prescription opioids: Regional variation and socioeconomic status - evidence from primary care in England. Int J Drug Policy 2019;64:87-94.

41. Safdar B, Heins A, Homel P, et al. Pain and Emergency Medicine Initiative Study Group. Impact of physician and patient gender on pain management in the emergency department-a multicenter study. Pain Med 2009;10:364-372.

42. Dumitrescu L, Ritchie MD, Brown-Gentry K, et al. Assessing the accuracy of observer-reported ancestry in a biorepository linked to electronic medical records. Genet Med 2010;12:648-650.

43. Hall JB, Dumitrescu L, Dilks HH, Crawford DC, Bush WS. Accuracy of administratively-assigned ancestry for diverse populations in an electronic medical record-linked biobank. PLoS One 2014;9: e99161.

44. Palumbo SA, Adamson KM, Krishnamurthy S, et al. Assessment of probable opioid use disorder using electronic health record documentation. JAMA Netw Open 2020;3:e2015909. 